\title{
A New In Situ Neuronal Model for Cryo-ET
}

Joseph Kim, Sihui Yang, Jill Wildonger and Elizabeth Wright

University of Wisconsin - Madison, Madison, Wisconsin, United States

With current technological advancements in cryo-electron tomography, researchers have expanded their projects in the past few years to investigate new specimens. Neuroscience in particular has greatly benefited from cryo-ET since studies first began nearly 15 years ago [1]. By culturing primary neurons onto EM grids for plunge-freezing, localization of subcellular regions and neuronal proteins can be investigated at nanometer-resolution. Such studies, when combined with cryo-CLEM, cryo FIB-SEM, and sub-tomogram averaging, have advanced our understanding of synapses, neurodegeneration, and axonal transport [2-4]. However, all cryo-ET studies using primary cultured neurons have been derived from embryonic mice or rats. Many more discoveries in neuroscience can be made by cryo-ET if we diversify our model selection. One potential model is Drosophila melanogaster, also known as the common fruit fly, which researchers have used to make substantial contributions to the field of neuroscience for the last 100 years. Such discoveries involving fruit flies include cloning the first reported voltage-dependent potassium channel and identifying pathways in growth cone guidance and neuronal migration [5]. However, to translate these studies to the ultrastructural level, a new method is needed to obtain wellpreserved, near-native neuronal samples from the fruit fly.

Here, we present a new primary neuronal culturing platform on EM grids, larval brains from the fruit fly [6]. Fruit flies are a suitable model organism in that they are simple and inexpensive to maintain, have a large number of offspring in a short time interval, have well annotated genomics and proteomics, and are easy to genetically manipulate. Neuron cell biology is also greatly conserved in flies and vertebrate neurons, allowing translatable inferences to be made to vertebrate models while studying fly neurons. With concanavalin A as the optimal extracellular matrix (ECM) for grid-coating and good culturing technique, we found that fruit fly larval neurons were well-adhered and dispersed when grown on carboncoated gold EM grids. Through pan-neuronally expressed GFP, we can visualize neuron specific tissue by with fluorescent microscopy and cryo-CLEM [7]. We show well-extended neurites were formed for each neuron adhered to the grid after only 2-3 days in vitro. We determined that single-sided blotting from the backside during plunge-freezing produced grids where neurons were well preserved in vitreous ice with thin neurites that can be imaged by cryo-EM. Cryo-EM images of these neurites show linear microtubule structures and organelle transport, making fruit fly neurons potentially suitable for ultrastructural studies of microtubules and axonal transport. Because of their rapid growth rate, fruit fly neurons can be a potential model for cryo-ET studies where high-throughput is required.

The genetic tool box in flies makes manipulation and visualization of a specific neuron or a group of neurons possible. Taking advantage of this, future work in flies could look at different neuron types of interest, for instance, a motor neuron versus a sensory neuron [8]. Maskless photopatterning, in combination with concanavalin A for ECM deposition, will also be used for neuronal placement at a spatially confined region to increase throughput of cryo-ET and correlative imaging workflows [9]. This will ensure that neurons from fruit fly larvae can be used cryo-ET studies with optimized versatility, costeffectiveness, and throughput [10]. 


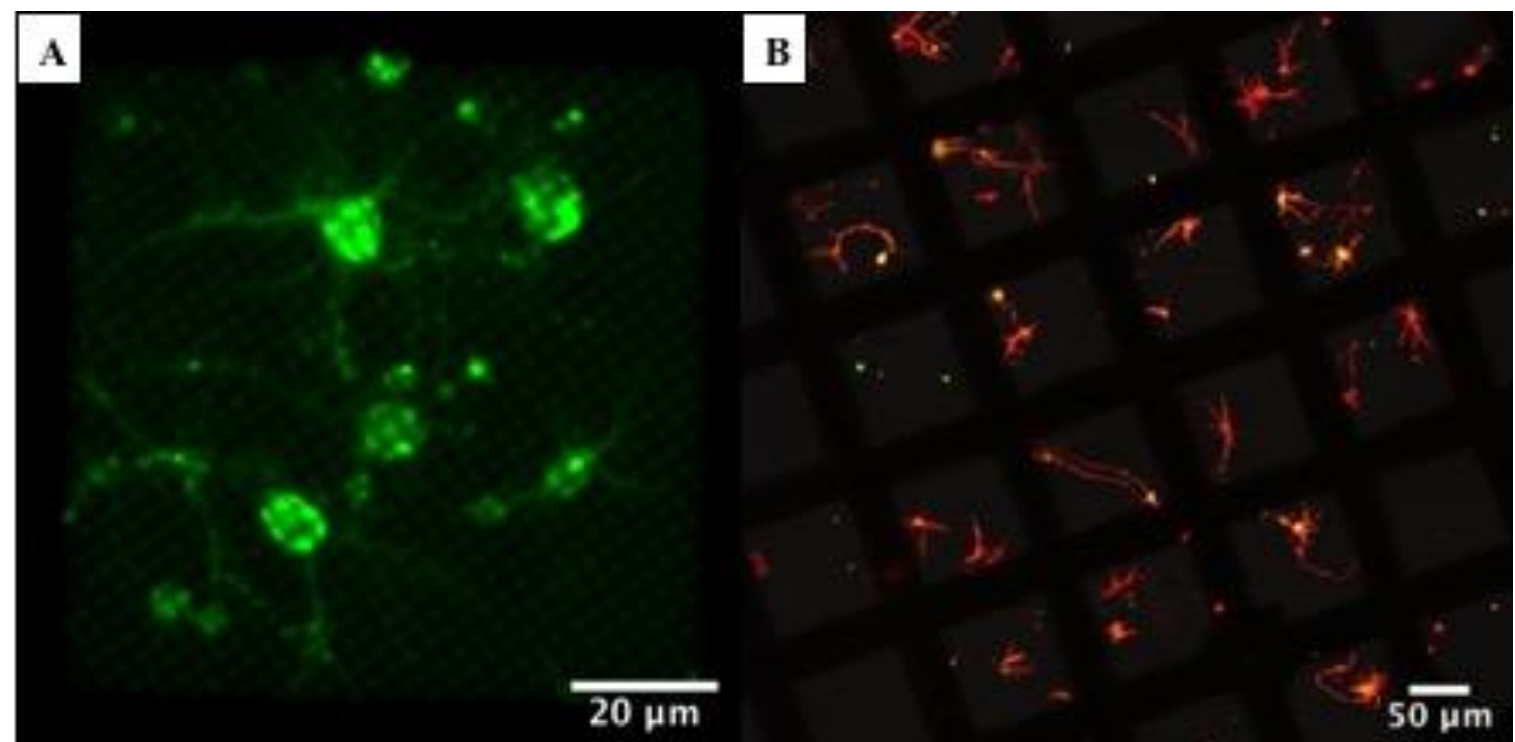

Figure 1. Figure 1. (A) Light microscopy images of cultured fruit fly neurons on gold Quantifoil 200 mesh R 2/1 grids, imaged after three days. The neurons were cultured from isolated brains from 3rd instar larvae that express a transgenic membrane marker mCD4::GFP under the pan-neuronal driver elav. (B) Fruit fly neurons stained for ELAV (green) and by horse-radish peroxidase (red), with overlapping fluorescent signals appearing as yellow.

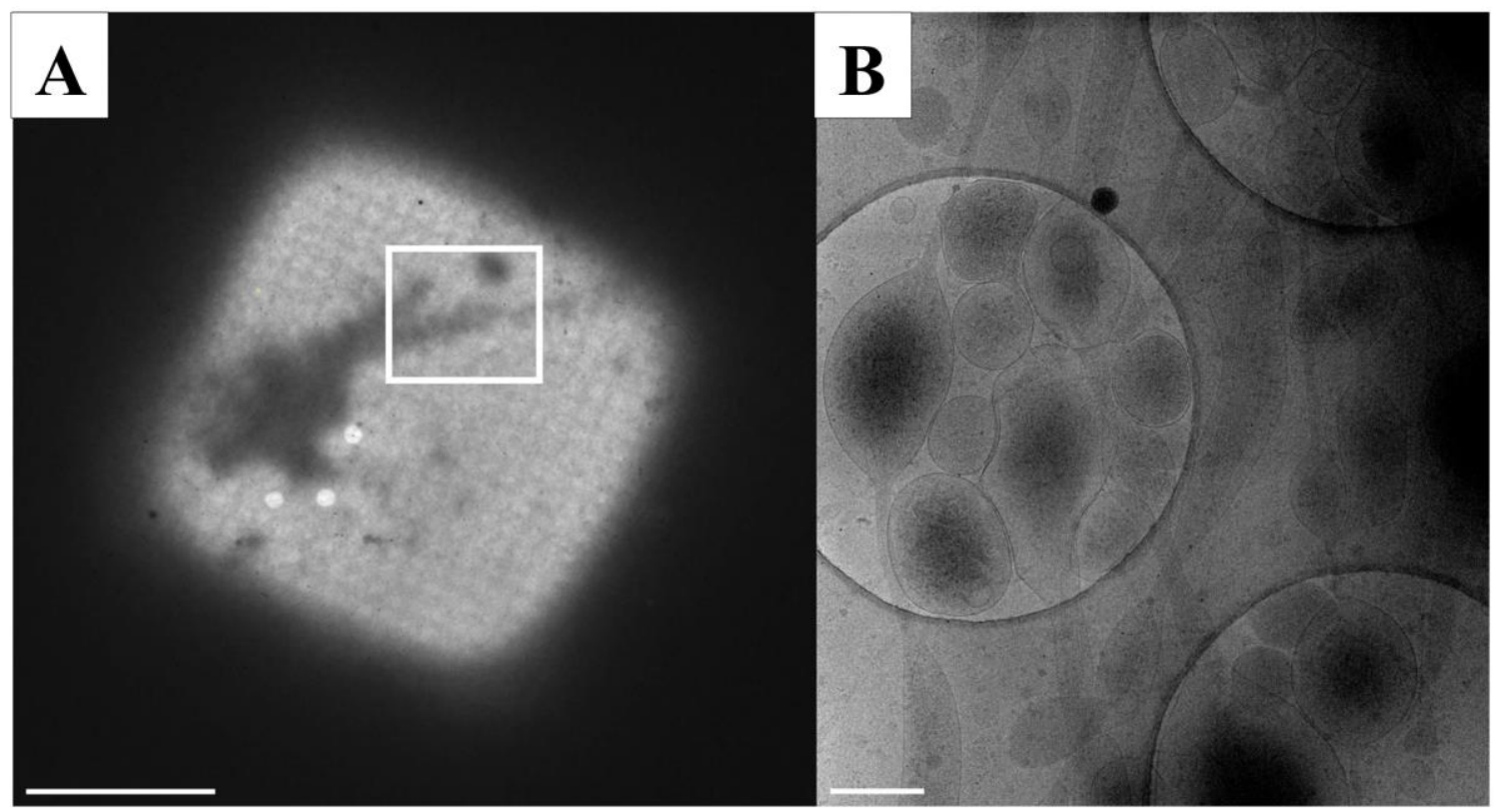

Figure 2. Figure 2. Cryo-EM images of vitrified fruit fly neurons. (A) Low-magnification image of frozen neuron, where the neurites are well-preserved (white box). (B) High-magnification image of neurite from (A). Cytoskeletal features and membranes are well-visualized, which indicates good preservation. Scale bar is $25 \mu \mathrm{m}$ for (A) and $500 \mathrm{~nm}$ for (B). 
References

1. Garvalov, B. K. et al. Luminal particles within cellular microtubules. J. Cell Biol. 174, 759-765 (2006). 2. Tao, C.-L. et al. Differentiation and Characterization of Excitatory and Inhibitory Synapses by Cryoelectron Tomography and Correlative Microscopy. J. Neurosci. 38, 1493-1510 (2018).

3. Guo, Q. et al. In Situ Structure of Neuronal C9orf72 Poly-GA Aggregates Reveals Proteasome Recruitment. Cell 172, 696-705.e12 (2018).

4. Fischer, T. D., Dash, P. K., Liu, J. \& Waxham, M. N. Morphology of mitochondria in spatially restricted axons revealed by cryo-electron tomography. PLOS Biology 16, e2006169 (2018).

5. Bellen, H. J., Tong, C. \& Tsuda, H. 100 years of Drosophila research and its impact on vertebrate neuroscience: a history lesson for the future. Nat Rev Neurosci 11, 514-522 (2010).

6. Egger, B., van Giesen, L., Moraru, M. \& Sprecher, S. G. In vitro imaging of primary neural cell culture from Drosophila. Nature Protocols 8, 958-965 (2013).

7. Duffy, J. B. GAL4 system in Drosophila: a fly geneticist's Swiss army knife. Genesis (2002)

8. Sanyal, S. Genomic mapping and expression patterns of C380, OK6 and D42 enhancer trap lines in the larval nervous system of Drosophila. Gene Expression Patterns 9, 371-380 (2009).

9. Toro-Nahuelpan, M. et al. Tailoring cryo-electron microscopy grids by photo-micropatterning for incell structural studies. Nature Methods 17, 50-54 (2020).

10. This research was supported in part by funds from the University of Wisconsin, Madison, the Burroughs Wellcome Fund (1018803.01), and National Institutes of Health (R01GM104540-03S1) to E.R.W. The authors gratefully acknowledge use of facilities and instrumentation at the UW-Madison Wisconsin Centers for Nanoscale Technology (wisc.edu) partially supported by the NSF through the University of Wisconsin Materials Research Science and Engineering Center (DMR-1720415). 